\title{
Handbuch Kulturportale
}

Online-Angebote aus Kultur und Wissenschaft

Herausgegeben von

Ellen Euler, Monika Hagedorn-Saupe, Gerald Maier, Werner Schweibenz und Jörn Sieglerschmidt 
ISBN 978-3-11-040571-2

e-ISBN (PDF) 978-3-11-040577-4

e-ISBN (EPUB) 978-3-11-040580-4

\section{Library of Congress Cataloging-in-Publication Data}

A CIP catalog record for this book has been applied for at the Library of Congress.

\section{Bibliografische Information der Deutschen Nationalbibliothek}

Die Deutsche Nationalbibliothek verzeichnet diese Publikation in der

Deutschen Nationalbibliografie; detaillierte bibliografische Daten

sind im Internet über http://dnb.dnb.de abrufbar.

(c) 2015 Walter de Gruyter GmbH, Berlin/Boston

Umschlagabbildung: Artur Marciniec / iStock / Thinkstock

Satz: Michael Peschke, Berlin

Druck und Bindung: $\mathrm{CPI}$ books $\mathrm{GmbH}$, Leck

$\otimes$ Gedruckt auf säurefreiem Papier

Printed in Germany

www.degruyter.com 


\section{Inhalt}

\section{Teil 1 Einführung und Bedeutung von Kulturportalen}

Ellen Euler, Monika Hagedorn-Saupe, Gerald Maier, Werner Schweibenz, Jörn

Sieglerschmidt

Kulturportale im Web

Eine Einführung - 3

Jörn Sieglerschmidt

Wissensordnungen im analogen und im digitalen Zeitalter -12

\section{Teil 2 Rechtliche und wirtschaftliche Aspekte oder Aspekte der} Verwertung

Artur-Axel Wandtke

Kulturportale und Urheberrecht -33

Andrea Hänger

Gebührenordnungen im Widerspruch zu Informationsweiterverwendungsgesetz und Open Access?! - 44

John H. Weitzmann

Creative Commons für Kulturinstitutionen -51

Paul Klimpel

Eigentum an Metadaten?

Urheberrechtliche Aspekte von Bestandsinformationen und ihre Freigabe - $\mathbf{5 7}$

Uwe K. Schneider

Zwischen Datenschutz und Nutzeroptimierung — 65

Ellen Euler

Open Access - Verpflichtung oder Geschäftsmodell für Kultureinrichtungen?! — 81

Christian Kircher

Alles Offen. Alles Frei. Alles Gratis.

Open-Data als Impuls für einen Strukturwandel im Unternehmen Museum — 102 
Holger Simon

Cultural Entrepreneurship - Geschäftsmodelle für Kunst und Kultur 110

\section{Teil 3 Überregionale Portale}

Henning Scholz

Europeana - Digitale Dienstleistungs-Infrastruktur für Europas Kulturerbe - 125

Frank Frischmuth

Die Deutsche Digitale Bibliothek als nationales Kulturportal für

Deutschland — 133

Reinhard Altenhöner, Uwe Müller

Die Serviceplattform der Deutschen Digitalen Bibliothek — 147

Werner Schweibenz

BAM als überregionales Kulturportal für Deutschland

Ein Rückblick — 164

Irene Hyna, Bianca Pospischek

Kulturpool.at als nationales Kultur-Portal für Österreich — 173

Gerald Maier, Christina Wolf

Das Archivportal-D

Neue Zugangswege zu Archivgut innerhalb der Deutschen Digitalen

Bibliothek — 180

Susanne Waidmann

Das Europäische Archivportal — 191

David Kleingers

filmportal.de - Die zentrale Internetplattform zum deutschen Film — 204

Karl Heinz

Monasterium.Net - Europas virtuelles Urkundenarchiv — 215

Lisa Dieckmann

prometheus - das verteilte digitale Bildarchiv für Forschung \& Lehre e. V. -223 


\section{Teil 4 Regionale Portale}

Frank von Hagel

SPK-digital als Institutionsportal -233

Gisela Schulte-Dornberg

Das Digitale Kunst- und Kulturarchiv Düsseldorf (d:kult) -243

Gerda Koch

Europeana-Local-Österreich -252

Christoph Stuehn

Memobase - das Informationsportal zum audiovisuellen Kulturerbe der

Schweiz -260

Elisabeth Bracun, Monika Hagedorn-Saupe

Europeana Fashion -268

Thorsten Siegmann

Europeana 1914-1918

Unbekannte Geschichten und offizielle Dokumente zum Ersten Weltkrieg — 277

Daniel Fähle, Wolfgang Krauth

LEO-BW - Landeskundliches Informationssystem Baden-Württemberg — 284

Klaus Ceynowa, Stephan Kellner

Das bayerische Kulturportal bavarikon - digital, vernetzt, spartenübergreifend -292

Frank Dührkohp

Spartenübergreifende Präsentation von Kulturobjekten - das Landesportal Kulturerbe Niedersachsen -301

Kathrin Pilger

www.archive.nrw.de - Das Archivportal für Nordrhein-Westfalen — 308

Frauke Rehder

digiCULT - mehr als ein regionales Museumsportal — 314

Susanne Kopp-Sievers, Bettina Scheeder, Jens Wübbenhorst museum-digital - Ein zivilgesellschaftliches Projekt großer und kleiner Museen -322 
VIII

Inhalt

Marcus Weidner

Das Internet-Portal „Westfälische Geschichte“ — 330

Autoren -338

Register - 351 


\title{
Ellen Euler
}

\section{Open Access - Verpflichtung oder Geschäftsmodell für Kultureinrichtungen?!}

\section{Einführung}

Open Access (= engl. offener Zugang) steht als Glaubenssatz quasi vor der Klammer und wird vom europäischen Gesetzgeber als scheinbar alternativloses Diktum vorgegeben. Nicht mehr nur die öffentliche Verwaltung, sondern auch bestimmte kulturelle öffentliche Einrichtungen (Bibliotheken, Museen und Archive) sind nach dem Willen des europäischen Gesetzgebers seit dem 26. Juni 2013 gehalten, die Weiterverwendung ihrer Dokumente und Informationen unter bestimmten Voraussetzungen zu gestatten. Vor diesem Hintergrund stellt sich die grundsätzliche Frage, welchen Spielraum die Umsetzung der europäischen Vorgaben durch den nationalen Gesetzgebers den Kultureinrichtungen lässt, bestehende Geschäftsmodelle fortzusetzen. Möglicherweise sind diese dem Open Access-Gedanken verpflichtend anzupassen oder brechen gar völlig weg.

Der Frage, wo gesetzliche Vorgaben zu Open Access verpflichten, wo alte Geschäftsmodelle wegbrechen, wo Spielraum besteht und wo Open Access möglicherweise ein sinnvolles Geschäftsmodell ist, soll im Folgenden für die unterschiedlichen Kultur- und Wissenschaftseinrichtungen nachgegangen werden. Im Ergebnis ist dies, wie Thomas Dreier in einer Keynote zutreffend festgestellt hat, eine Frage nach Verteilungsgerechtigkeit:

\begin{abstract}
Wer soll aus den mit öffentlichen Mitteln finanzierten Informationsbeständen Gewinne erzielen dürfen? Es mag ja zutreffen, dass Informationen der öffentlichen Hand eine besonders verlässliche und wirtschaftlich wertvolle Ressource darstellen, auf der sich innovative Produkte und Dienstleistungen aufsetzen lassen, die der Wissensgesellschaft und mithin der Allgemeinheit förderlich sind. Aber ist es gerecht, dass Daten, die mit öffentlichen Mitteln gesammelt, generiert, vorgehalten und archiviert werden, nur von denjenigen wirtschaftlich genutzt werden können, die hierzu die wirtschaftlichen Voraussetzungen mitbringen? Handelt es sich möglicherweise um Klientelgesetzgebung im Deckmantel von Jedermann-Rechten? Läuft das Ganze am Ende nicht auf eine weitere Form der Sozialisierung von Verlusten und der Privatisierung von Gewinnen hinaus? Die Antwort auf diese Frage bedarf nicht allein der politischen bzw. normativen Wertvorgaben. Sie setzt vor allem - nicht leicht zu erlangende - Erkenntnisse darüber voraus, wie Wettbewerb und Innovation in den genannten Bereichen funktioniert und wie ein Marktversagen gegebenenfalls rechtlich zu korrigieren und eine Innovationsleistung bestmöglich zu fördern ist. ${ }^{1}$
\end{abstract}

1 Dreier, Eröffnungsrede auf Tagung „Kultur im Wandel. Informationen der öffentlichen Hand: Zugang und Nutzung." vom 1. März 2013 in Karlsruhe am ZKM. 


\section{Open Access - Was heißt das genau?}

Die Diskussion zu „Open Access“ wird auf unterschiedlichen Ebenen geführt.

Im engeren Sinne ist darunter der freie, im Sinne von „kostenlose, Zugang zu den von Seiten der Wissenschaftler im öffentlichen Auftrag und Dienst erschaffenen Publikationen“ gemeint. Das soll den wissenschaftlichen Diskurs mithilfe der digitalen und vernetzten Medien optimieren. Die öffentliche Hand verspricht sich darüber hinaus zugleich Einspareffekte und mehr Unabhängigkeit von den übermächtigen Wissenschaftsverlagen. Die Verlage halten mit dem Argument dagegen, dass sich private Investitionen in die Aufbereitung von Manuskripten und in die nachhaltige Sicherung von Veröffentlichungen lohnen müssen. Im Publikationswesen werden verschiedene Open-Access-Publikationswege unterschieden. Während beim goldenen Weg direkt in Open-Access-Verlagen und Zeitschriften veröffentlicht wird, wird beim grünen Weg die Speicherung bereits publizierter, begutachteter Fachartikel auf institutionellen oder fachlichen Dokumentenservern von Hochschulen oder Forschungsorganisationen veröffentlicht. Zur Verwirklichung von Open Access durch die Autoren und Stärkung von deren Rechten wurde jüngst mit der Normierung des Zweitveröffentlichungsrechts in $\S 38$ Abs. 4 Urheberrechtsgesetz (UrhG) eine Minimallösung umgesetzt. ${ }^{2}$

Im weiteren Sinne, und diese Auslegung soll für den vorliegenden Beitrag gelten, meint Open Access:

nicht nur den Zugang als passive Form der Rezeption, sondern Open Access gewährt auch Rechte des Fortschreibens und Bearbeitens. Der Rezipient darf auch zum Schöpfer im urheberrechtlichen Sinne werden, ${ }^{3}$

Open Access ist das Schlagwort für einen freien medientypübergreifenden Zugang $\mathrm{zu}$ wissenschaftlicher und kultureller Information über das Internet, also von Jedermann, zu jeder Zeit von jedem beliebigen Ort aus. Gleichzeitig ermöglicht eine offene Lizenzierung (z. B. mit Creative-Commons-Lizenzen ${ }^{4}$ ) die Anknüpfung und Nutzung. Hierfür ist erforderlich, dass freier Zugang nicht nur zu den Inhalten selbst, sondern auch zu den diese Inhalte erschließenden Daten, besteht.

Die von der öffentlichen Hand getragenen kulturellen und wissenschaftlichen Einrichtungen verfügen über digitale Angebote, anders ausgedrückt: „Datenschätze“, der unterschiedlichsten Art. Diese lassen sich unterteilen in Inhalte und Informationen zu den Inhalten (Metadaten).

2 Bruch, Christoph / Plüger, Thomas, Das Zweitveröffentlichungsrecht des § 38 Abs. 4 UrhG - Möglichkeiten und Grenzen bei der Anwendung in der Praxis, in ZUM 2014 S. 388 ff.

3 P. Suber, Gratis and Libre Open Access. From the SPARC Open Access Newsletter, August 2008, http://www.sparc.arl.org/resource/gratis-and-libre-open-access (letzter Aufruf 10. April 2015).

4 siehe: www.creativecommons.org (letzter Aufruf 10 April 2015). 


\section{$\mathrm{Zu}$ den Inhalten zählen neben}

- Schriftgut aus Wissenschaftseinrichtungen und Bibliotheken,

- Abbildungen von Werken der bildenden Kunst, Skulptur, Noten, Münzen und sonstigen Sammlungsgegenständen aus Museen,

- Abbildungen von Architektur und Baudenkmälern aus Einrichtungen der Denkmalpflege,

- Abbildungen von Archivgut aus den Archiven,

- Filmmaterial aus den Mediatheken,

- Tondokumente aus den Rundfunkarchiven.

$\mathrm{Zu}$ den Informationen zu den Inhalten zählen vor allem die Erschließungs- bzw. Beschreibungsinformationen, die umso wertvoller sind, je mehr Kontextinformation sie enthalten. Das gilt angesichts des wachsenden Einsatzes mobiler Endgeräte etwa für Daten mit Raumbezug, aus denen Umgebungsinformationen generiert werden können. Ebenso wichtig sind in diesem Zusammenhang Strukturdaten, mit denen verknüpft sich eineindeutige Aussagen zu Entitäten wie Personen und Orten treffen lassen und Raumbezüge herstellen, und etwa Aussagen zum Standort einzelner Kultur- und Wissensschätze machen lassen.

\section{Verpflichtung zu Open Access?}

Kultur- und Wissenschaftseinrichtungen setzen Open Access unterschiedlich um (hierzu siehe im Folgenden Status Quo). Es fragt sich jedoch, ob gesetzliche Rahmenbedingungen einen bestimmten Umfang normieren und verbindlich vorgeben.

Die Europäische Kommission beschwört im Rahmen der von ihr angestoßenen europäischen Digitalisierungsinitiativen die Öffnung und Demokratisierung des europäischen kulturellen Erbes. ${ }^{5}$ Dabei betont sie sowohl die sozio-kulturelle Bedeutung von Informationen des kulturellen Sektors als auch das wirtschaftliche Potenzial derselben. ${ }^{6}$ Nachdem Studien zur Bedeutung der Öffnung öffentlicher Informationsbestände oder über die jährlichen Investitionen im Bereich der Informationsgenerierung, sowie über den Wert von Informationen, keinen anderen Schluss zulassen, als dass die Öffnung alternativlos vorangetrieben werden muss, ${ }^{7}$ wurde erstmals im Jahr

5 Vgl. Europäische Inhalten in globalen Netzen, 4 April 2001, 1; KOM (2005) 465 eng., i2010: Digitale Bibliotheken, 30.09.2005, 3-4.

6 Vgl. beispielhaft: KOM (2005) 465 eng., i2010: Digitale Bibliotheken, 30. September 2005, 5; SEK (2006) , Empfehlungen der Kommission, 24.08.2006, 2; KOM (2010) 245 endg., Mitteilung der Kommission an das Europäische Parlament, den Rat, den Europäischen Wirtschafts- und Sozialausschuss und den Ausschuss der Regionen, 10.

7 Vgl. u. a.: Commission of the European Communities, Publaw Subject Report, Januar 1991; Policy Studies Institute/Gesellschaft für Mathematik und Datenverarbeitung/Centre de Recherches Informatique et Droit/British Library Consultancy Service, Publaw II, Final Report Europe, 1993 und Policy Studies Institute/Centre de Recherches Informatique et Droit, Publaw Report III, November 1995 und 
2003 die Richtlinie über die Weiterverwendung von Informationen des öffentlichen Sektors (PSI-Richtlinie) verabschiedet. ${ }^{8}$ Dabei soll die Weiterverwendung frei zugänglich zu machender Informationen sowohl zu kommerziellen als auch zu nicht-kommerziellen Zwecken gestattet sein. Im Wesentlichen geht es um die Umsetzung des Gleichheitsgrundsatzes sowie von Transparenzvorgaben für öffentliche Stellen. Insbesondere Unternehmen sollen hierdurch in die Lage versetzt werden, das Potenzial dieser Informationen - etwa für elektronische Mehrwertdienste - auszuschöpfen, um so zu Wirtschaftswachstum und zusätzlichen Arbeitsplätzen beizutragen. Laut den Erwägungsgründen zielt die Richtlinie ausdrücklich nicht nur auf die Stärkung der Wirtschaft ab, sondern vor allem auf die Förderung von Transparenz und Demokratie innerhalb Europas. ${ }^{9}$ Im Jahr 2013 wurde die Richtlinie aus dem Jahr 2003 geändert und der Anwendungsbereich um bestimmte kulturelle Einrichtungen (Bibliotheken, Museen und Archive) erweitert. ${ }^{10}$

Die PSI-Richtlinie von 2003 wurde in Deutschland im Gesetz zur Informationsweiterverwendung (IWG) im Jahr 2006 umgesetzt, in das auch die Änderungen aus der Richtlinie von 2013 eingearbeitet wurden. Das entsprechende Gesetz wurde fristgemäß verabschiedet. ${ }^{11}$ Leider finden sich die klugen Anmerkungen des Bundesrates, die in der Stellungnahme vom 27. März 2015 zum Entwurf der Bundesregierung vom 13. Februar 2015 enthalten waren, in der finalen und am 7. Mai 2015 verabschiedeten Gesetzesfassung und Begründung vom 15. April 2015 nicht wieder. In seiner Stellungnahme weist der Bundesrat zu Recht darauf hin, dass der Entwurf zum IWG in $\S 8$ unzureichend ist, wenn dort nur von einem nationalen Datenportal für mit Metadaten versehene Daten (Informationen) im Sinne von solchen der Verwaltung nach § 12 Abs. 1 EGovG die Rede ist. Diese Funktion erfüllt das nationale Datenportal GovData. Vielmehr muss dort auch das nationale Datenportal für mit Metadaten versehene Daten und Objekte (Informationen im Sinne des IWG) aus den erstmals, neben sonstigen öffentlichen Stellen, in den Anwendungsbereich des IWG einbezogenen Kultur-

Vgl. Pira International, Commercial Exploitation of Europe‘s public sector information, 30. Oktober 2000. Das Potenzial der Veredelung von Daten für das Wirtschaftswachstum offener Verwaltungsdaten wird allein in Europa auf 40 Milliarden Euro jährlich geschätzt, gepaart mit offenen Daten aus anderen Quellen steigt das Potenzial auf 140 Milliarden Euro pro Jahr. Siehe zusammenfassend: Vickery, J., Review of Recent PSI Re-Use Studies, im Auftrag der Europäischen Kommission 2011. Siehe: http://ec.europa.eu/digital-agenda/en/news/economic-analysis-psi-impacts (letzter Abruf: 23. Februar 2015).

8 RL 2003/98/EG. Zur Entwicklung der PSI-Richtlinie siehe: H. Wirtz, DuD 2014, 389 ff.

9 Vgl. Erwägungsgründe 2, 5 und 16 der ursprünglichen PSI-Richtlinie.

10 RL 2013/37/EU. Gemäß Erwägungsgrund 18 verbleiben „Andere kulturelle Einrichtungen (wie Orchester, Opern, Ballette sowie Theater), einschließlich der zu diesen Einrichtungen gehörenden Archive, [...] auch weiterhin außerhalb des Anwendungsbereiches [...], zumal es sich in diesen besonderen Fällen um „darstellende Künste“ handelt.“ Siehe zum Anwendungsbereich ausführlich H. Wirtz in: E. Euler / P. Klimpel, Der Vergangenheit eine Zukunft, Berlin 2015, $260 \mathrm{ff}$.

11 Zum Gesetzgebungsverfahren und den Drucksachen siehe: http://dipbt.bundestag.de/extrakt/ ba/WP18/651/65153.html (11. Mai 2015). 
und Wissenschaftseinrichtungen benannt sein: Die Deutsche Digitale Bibliothek (DDB). Auch Artikel 9 der Richtlinie (RL) 2013/37/EU, der praktische Vorkehrungen vorsieht, welche die Suche nach bzw. in zur Weiterverwendung zur Verfügung zu stellender Dokumente von in den Anwendungsbereich einbezogenen Einrichtungen erleichtern sollen, spricht von Internet(daten)portalen im Plural. Während GovData, die standardisierte Bereitstellung offener Verwaltungsdaten durch Bund, Länder und Kommunen koordiniert, koordiniert die DDB die standardisierte Bereitstellung offener Kulturdaten durch Bund, Länder und Kommunen und stellt diese über eine Programmierschnittstelle zur Verfügung.

Nicht nur für den Verwaltungssektor (mit GovData), sondern auch für den kulturellen Sektor (mit DDB), werden somit bereits praktische Vorkehrungen zur Erfüllung der Ziele aus der Richtlinie getroffen, obwohl in Umsetzung der RL 2013/37/EU öffentliche Bibliotheken, Museen und Archive erstmalig in den Geltungsbereich mit einbezogen werden.

Die Bundesregierung weist die Bemerkung und Ergänzungsbitte des Bundesrates in ihrer Antwort auf die Stellungnahme des Bundesrates mit der Begründung zurück, dass $\S 8$ IWG sich lediglich auf Metadaten (Datenbeschreibungen) von Daten im Sinne des $\S 12$ Abs. 1 EGovG, nicht aber auf die eigentlich weiterzuverwendenden Informationen beziehe und dass die DDB keine solchen Daten besitze und auch kein Metadatenportal sei. Das ist jedoch in doppelter Hinsicht zu kurz gegriffen: Zum einen genügt § 8 IWG dann, wenn er sich lediglich auf Metadaten im Sinne des EGovG bezieht, nicht den Voraussetzungen aus Art. 9 der Richtlinie von 2013, zum anderen stimmt auch die Schlussfolgerung nicht, dass die DDB keine Metadaten enthalte. Natürlich enthält die DDB Metadaten! Diese stellt sie über ihre Programmierschnittstelle frei zur Verfügung und erfüllt damit genau die von Art. 9 der europäischen Richtlinie von 2013 geforderte Funktion für den Kulturbereich.In diesem Jahr findet in Berlin zum zweiten Mal der Kulturhackathon „Coding da Vinci“ als Kooperationsprojekt der Digitalisierungsstelle Berlin (digis); Wikimedia Deutschland, der Open Knowledge Foundation (OKF) Deutschland und der Deutschen Digitalen Bibliothek statt, bei dem Kulturinstitutionen mit der Entwickler-, Designer- und Gamescommunity ins Gespräch kommen und aus frei nutzbaren Kulturdaten neue Anwendungen, mobile Apps, Dienste, Spiele und Visualisierungen umsetzen..$^{12} \mathrm{Im}$ Vorjahr sind auf der Anwendung der frei nutzbaren und über Schnittstellen standardisiert abrufbaren Kulturdaten spannende Anwendungen entstanden und haben einen ersten Eindruck von dem Potenzial der Kulturdaten vermittelt.

12 siehe: http://codingdavinci.de/ (11. Mai 2015). 


\section{Anwendungsbereich und Vorgaben aus IWG:}

Vier Voraussetzungen müssen kumulierend vorliegen und sind jeweils im Einzelfall zu prüfen, damit ein Anspruch auf Weiterverwendung von Informationen der öffentlichen Hand aus dem IWG besteht: Erstens muss sich der Anspruch gegen eine Einrichtung des öffentlichen Rechts ${ }^{13}$ richten, zweitens muss zu der angefragten Information ein uneingeschränktes Zugangsrecht aus anderen Gesetzen bestehen, drittens müssen die angefragten Informationen im öffentlichen Auftrag angefertigt oder in den Bestand übernommen ${ }^{14}$ worden sein und viertens müssen sie sich im Besitz ${ }^{15}$ der Einrichtung befinden.

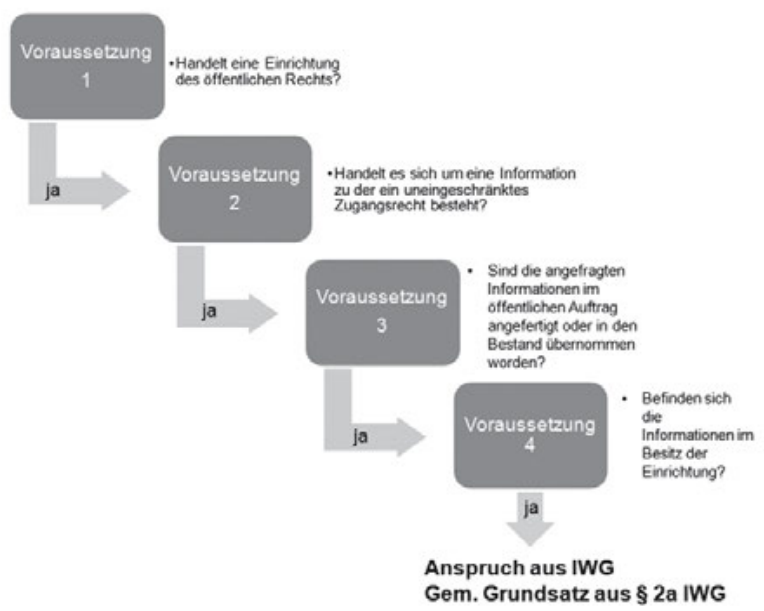

Abb. 1: Anspruch aus dem IWG (Grafik von Ellen Euler)

$\mathrm{Zu}$ 1:

Eine Einrichtung ist dann eine Einrichtung des öffentlichen Rechts, wenn sie zu dem besonderen Zweck gegründet wurde, eine im Allgemeininteresse liegende Aufgabe nicht gewerblicher Art zu erfüllen, Rechtspersönlichkeit besitzt und überwiegend von öffentlichen Stellen finanziert wird oder hinsichtlich ihrer Leitung einer öffentlichen Stelle unterliegt oder ein Verwaltungs-, Leitungs-, oder Aufsichtsorgan hat, das mehrheitlich aus Mitgliedern besteht, die von öffentlichen Stellen ernannt worden sind. ${ }^{16}$ Entsprechend siehe $\S 2$ Abs. 1 lit b IWG.

13 entsprechend § 2 Abs. 1 lit b IWG

14 ansonsten ist der Anwendungsbereich nicht eröffnet. Siehe entsprechend §1 Abs. 2 Nr. 3 IWG 15 Das bedeutet, die Einrichtung muss zur Gestattung der Weiterverwendung befugt sein.

16 Die Begriffsbestimmungen „öffentliche Stelle“ und „Einrichtung des öffentlichen Rechts“ sind den Richtlinien über das öffentliche Auftragswesen entnommen (Richtlinien 92/50/EWG (1), 93/36/ EWG (2), 93/37/EWG (3) und 98/4/EG (4)). Entsprechend siehe § 2 Abs. 1 lit b IWG. 


\section{$\mathrm{Zu} 2:$}

Das IWG regelt nicht den Zugang zu Informationen, sondern baut auf den bestehenden Regelungen (z. B. Informationsfreiheits- und Umweltinformationsgesetze des Bundes und der Länder) auf. Ein Recht auf Weiterverwendung von Informationen der öffentlichen Hand besteht nur, sofern diese der Öffentlichkeit, z. B. aufgrund des Informationsfreiheitsgesetzes, zur Verfügung gestellt werden.

\section{$\mathrm{Zu}$ 3:}

Unabhängig von dem jeweiligen Speichermedium umfasst der Begriff des „Dokuments“ jede Darstellung von Handlungen, Tatsachen oder Informationen. ${ }^{17}$ Information, die unter den Anwendungsbereich des IWG fällt, ist gem. § 2 Nr. 2 IWG ,jede Aufzeichnung auf elektronischen oder nichtelektronischen Datenträgern.“

Selbst wenn eine Bibliothek, ein Archiv oder ein Museum als öffentliche Einrichtung im Sinne der Richtlinie zu qualifizieren ist, bedeutet dies nicht zwangsläufig, dass jegliche Informationen, über die eine solche Einrichtung verfügt, unter die Bestimmungen der PSI-Richtlinie fallen. So sind gemäß Artikel 1 Abs. 2 lit. a der PSI-Richtlinie solche Dokumente ausgenommen, deren Bereitstellung nicht unter den öffentlichen Auftrag der Einrichtung zu fassen ist. Gemäß Art. 1 Nr. 2 lit. b sind Dokumente ausgeschlossen, die im geistigen Eigentum Dritter stehen, wobei dies laut Erwägungsgrund 22 der Richtlinie allein Urheberrechte und verwandte Schutzrechte Dritter umfasst.

$\mathrm{Ob}$ die Erstellung einer Information bzw. ihre Übernahme in den Bestand zum Auftrag der öffentlichen Einrichtung zählt, ist in Deutschland mangels einer verbindlichen Festlegung des öffentlichen Auftrags von Museen und Bibliotheken für diese jeweils im Einzelfall zu prüfen. Allein für öffentliche Archive wird der öffentliche Auftrag durch die Archivgesetze der Länder und des Bundes definiert. ${ }^{18}$

$\mathrm{Zu}$ 4:

Eine öffentliche Stelle besitzt eine Information, wenn sie berechtigt ist, die Weiterverwendung zu genehmigen. Auf etwaige Eigentumsverhältnisse kommt es dagegen nicht an.

Kulturelle Einrichtungen sind nicht den gleichen strengen Voraussetzungen der PSI-Richtlinie unterworfen, wie die sonstigen unterworfenen Einrichtungen. Ihre Einbeziehung ist zwar mit einer Reihe von Pflichten verbunden, gleichzeitig wurde jedoch durch die Einführung bestimmter Privilegien und Erleichterungen der Sonderrolle der Kultureinrichtungen genüge getan. So wird nur bei gemeinfreien Inhalten, also solchen an denen Urheberrechte und sonstige Schutzrechte nicht mehr beste-

17 Vgl. Artikel 2 Nr. 3 lit a und Erwägungsgrund 11 der PSI-Richtlinie.

18 E. Euler, Das kulturelle Gedächtnis im Zeitalter digitaler und vernetzter Medien und sein Recht, Bad Honnef 2011, 81. Der öffentliche Auftrag für Archive besteht in der Übernahme, Erfassung, Verwahrung, Bewertung, Sicherung, Bereitstellung und Veröffentlichung von Archivgut. 
hen, das bisherige Verwertungsmonopol der besitzenden Einrichtungen aufgelöst. Dagegen besteht grundsätzlich keine Pflicht zur Gestattung der Weiterverwendung für solche Inhalte, für die die Einrichtungen die Rechte innehaben. Hier behalten die Einrichtungen die Entscheidungshoheit über die Gestattung zur Weiterverwendung. Wenn die Einrichtungen die Weiterverwendung gestatten, dann ist diese jedoch unter denselben Voraussetzungen zu gestatten, wie die Gestattung zur Weiterverwendung an Inhalten, an denen keine Rechte bestehen. Geplant ist nach dem Gesetzentwurf vom 15. April 2015 nach § 2 IWG folgenden § 2a einzufügen:

\begin{abstract}
§ 2a Grundsatz der Weiterverwendung
Informationen, die in den Anwendungsbereich dieses Gesetzes fallen, dürfen weiterverwendet werden. Für Informationen, an denen Bibliotheken, einschließlich Hochschulbibliotheken, Museen oder Archiven Urheber- oder verwandte Schutzrechte oder gewerbliche Schutzrechte zustehen, gilt dies nur, soweit deren Nutzung nach den für diese Schutzrechte geltenden Vorschriften zulässig ist oder die Einrichtung die Nutzung zugelassen hat; die Bedingungen der Nutzung müssen den Vorschriften dieses Gesetzes entsprechen.
\end{abstract}

Eine weitere Privilegierung ist die, dass kulturelle Einrichtungen gegenüber den sonstigen Einrichtungen, die in den Anwendungsbereich fallen, nicht darauf beschränkt sind, für die Weiterverwendung allein die sogenannten Grenzkosten ${ }^{19}$ geltend zu machen. ${ }^{20}$ Sie können bei der Gebührenberechnung die Kosten für Erfassung, Digitalisierung und Rechteklärung zuzüglich einer angemessenen Gewinnspanne berücksichtigen. Problematisch ist, dass es in den Verantwortungsbereich der kulturellen Einrichtungen fällt, die Angemessenheit ihrer verlangten Vergütungen gegebenenfalls nachweisen zu müssen. ${ }^{21}$

Um die Digitalisierung der kulturellen Informationen nicht zu behindern, werden Museen, Bibliotheken und Archive auch dahingehend privilegiert, dass sie für die Durchführung auch weiterhin private Partnerschaften (sog. public private partnerships) eingehen können. ${ }^{22}$

Wenn die in den Anwendungsbereich der PSI-Richtlinie und des IWG fallenden kulturellen Einrichtungen für bestimmte digitale Angebote verpflichtet sind, diese frei zur Weiterverwendung zur Verfügung zu stellen und ein entsprechendes Zugangs-

19 Damit sind die Kosten umfasst, die durch die Reproduktion, Bereitstellung und Weiterverbreitung entstanden sind. Vgl. Artikel 6 Abs. 1 der PSI-Richtlinie.

20 Museen, Bibliotheken und Archive sind nach Artikel 6 Abs. 2 lit. c der Richtlinie von der Beschränkung auf die Geltendmachung der Grenzkosten explizit ausgenommen.

21 Für Abbildungen künstlerischer Werke, die für die Kreativindustrie sicher besonders attraktiv sind, kann die Marktüblichkeit der eigenen Vergütungsansätze gut mit den jährlich von den Fotografieverbänden gemeinsam herausgegebenen „Übersicht der marktüblichen Vergütungen für Bildnutzungsrechte“ - der so genannten MFM-Liste belegt werden.

22 Vgl. Erwägungsgrund 30 der Änderungsrichtlinie zur PSI-Richtlinie RL 2013/37/EU. Zu den weiteren Vorgaben der RL siehe: H. Wirtz in: E. Euler / P. Klimpel, Der Vergangenheit eine Zukunft, Berlin 2015, 260 ff. (S. 274). 
recht existiert, bleibt diesen Einrichtungen möglicherweise keine andere Wahl, als ihr Geschäftsmodell umzustellen.

Die PSI-Richtlinie regelt die Grundsätze für die Berechnung von Entgelten in den Erwägungsgründen 22 bis 29 und Artikel 6, die fast wortgleich im Entwurf zum IWG vom 15. April 2015 umgesetzt wurden:

$\S 5$ Grundsätze zur Entgeltberechnung

(1) Entgelte für die Weiterverwendung von Informationen sind auf die Kosten beschränkt, die durch die Reproduktion, Bereitstellung und Weiterverbreitung verursacht werden.

(2) Absatz 1 ist nicht anzuwenden auf

1. öffentliche Stellen, die ausreichend Einnahmen erzielen müssen, um einen wesentlichen Teil ihrer Kosten im Zusammenhang mit der Erfüllung ihrer öffentlichen Aufträge zu decken; 2. Informationen, für die die betreffende öffentliche Stelle aufgrund von Rechtsvorschriften ausreichend Einnahmen erzielen muss, um einen wesentlichen Teil der Kosten im Zusammenhang mit ihrer Erfassung, Erstellung, Reproduktion und Verbreitung zu decken;

3. Bibliotheken, einschließlich Hochschulbibliotheken, Museen und Archive.

In den in Absatz 2 Nummern 1 und 2 genannten Fällen berechnen die betreffenden öffentlichen Stellen die Gesamtentgelte nach von ihnen festzulegenden objektiven, transparenten und nachprüfbaren Kriterien. Die Gesamteinnahmen dieser Stellen aus der Bereitstellung von Informationen und der Gestattung ihrer Weiterverwendung in dem entsprechenden Abrechnungszeitraum dürfen die Kosten ihrer Erfassung, Erstellung, Reproduktion und Verbreitung, zuzüglich einer angemessenen Gewinnspanne, nicht übersteigen. Die Entgelte werden unter Beachtung der für die betreffenden öffentlichen Stellen geltenden Buchführungsgrundsätze berechnet.

Wenn die in Absatz 2 Nummer 3 genannten öffentlichen Stellen Entgelte verlangen, dürfen die Gesamteinnahmen aus der Bereitstellung von Informationen und der Gestattung ihrer Weiterverwendung in dem entsprechenden Abrechnungszeitraum die Kosten ihrer Erfassung, Erstellung, Reproduktion, Verbreitung, Bewahrung und der Rechteklärung, zuzüglich einer angemessenen Gewinnspanne, nicht übersteigen. Die Entgelte werden unter Beachtung der für die betreffenden öffentlichen Stellen geltenden Buchführungsgrundsätze berechnet.“

Für die in den Anwendungsbereich von PSI-Richtlinie und IWG fallenden Einrichtungen ist insbesondere $\S 5$ Abs. 1, Abs. 2 Nr. 3 und Abs. 4 S. 1 interessant. Im Fazit sind diese Einrichtungen bei der Berechnung der Gebühren für die Gestattung zur Weiterverwendung ihrer digitalen Angebote nicht auf die sogenannten Grenzkosten (durch Reproduktion, Bereitstellung und Weiterverbreitung entstandene Kosten) beschränkt, sondern können eine angemessene Gewinnspanne auf diese Kosten aufschlagen. Die Gewinnspanne kann sich gemäß Erwägungsgrund 23 der Richtlinie an den im Privatsektor für die Weiterverwendung identischer oder ähnlicher Dokumente erhobenen Gewinnspannen orientieren. 


\section{Zwischenfazit}

Damit bleibt das gängige Geschäftsmodell der Gestattung zur Weiterverwendung / Nutzung durch Lizenzierung gegen Gebühr grundsätzlich weiterhin anwendbar. Weder die PSI-Richtlinie, noch das IWG in der vorliegenden Entwurfsfassung vom 15. April 2015, stehen dem Geschäftsmodell des Verkaufs des Produkts „Information oder Inhalt“ zur Erwirtschaftung von Umsatz entgegen. Die kulturellen Einrichtungen haben allerdings zu prüfen, ob ihre Gebührengrundsätze denen aus der PSI-Richtlinie und dem umgesetzten IWG entsprechen.

\section{Geschäftsmodelle kultureller Einrichtungen - Status Quo}

Zwar verwirklichen Kultur- und Wissenschaftseinrichtungen einen zumeist gesetzlichen Auftrag der kulturellen Daseinsfürsorge und haben kulturelle Teilhabe im 21. digitalen und vernetzten Zeitalter auch über das Internet zu ermöglichen, ${ }^{23}$ da die Digitalisierung und Ermöglichung kultureller Teilhabe über das Internet bis auf wenige Ausnahmen und, soweit nicht Drittmittel von der DFG oder anderer Stelle akquiriert werden können, aus dem vorhandenen Etat der Kultur- und Wissenschaftseinrichtungen zu leisten ist, sind sie trotz alledem vielfach gezwungen, über (Re-) Finanzierungsmodelle nachzudenken. Die Kultur- und Wissenschaftseinrichtungen sehen sich vor eine unlösbare Aufgabe gestellt: Wie kann einerseits für den Nutzer (kosten)freier Zugang zu Kultur und Wissen auch über das Internet gewährleistet werden und andererseits eine sprudelnde Einnahmequelle generiert werden?

Ausgangslage ist die, dass sich gegenwärtig über 500 Kultur- und Wissenschaftseinrichtungen zu Open Access formal bekennen, indem sie die „Berliner Erklärung über den offenen Zugang zu wissenschaftlichem Wissen“ unterzeichnet haben. ${ }^{24}$ Diese von der Max-Planck-Gesellschaft im Jahr 2003 initiierte Erklärung zu Open Access hat das Ziel, den freien und einfachen Zugang zu wissenschaftlichem Wissen und zum kulturellen Erbe zu fördern. Museen, Bibliotheken und Archive werden als die klassischen „Verwalter von kulturellem Erbe“ ausdrücklich aufgefordert, freien Zugang zu wissenschaftlichen Forschungsergebnissen, Metadaten, Quellenmaterial, Bildern, Grafiken und Multimedia-Materialien ,in jedem beliebigen digitalen Medium und für jeden verantwortbaren Zweck“ zu gestatten.

23 T. Dreier / E. Euler, Onleihe und virtueller Museumsbummel. Das Menschenrecht auf kulturelle Teilhaber im 21. Jahrhundert, in: P. Klimpel / E. Euler (Hrsg.), Der Vergangenheit eine Zukunft, 2015, $192 \mathrm{f}$.

24 http://openaccess.mpg.de/68053/Berliner_Erklaerung_dt_Version_07-2006.pdf (letzter Aufruf 10. April 2015). 
Die Berliner Erklärung wurde zunächst vor allem von Bibliotheken und Wissenschaftseinrichtungen unterschrieben. Mit der Unterzeichnung der Erklärung Ende 2013 durch die Stiftung Preußischer Kulturbesitz als große kulturelle Dachorganisation, die alle drei Sparten - Bibliotheken, Museen und Archive - umfasst, sollte der Damm gebrochen und auch Museen für die wichtige Aufgabe der Gewährung kultureller Teilhabe über das Internet sensibilisiert und motiviert werden. ${ }^{25}$ Soweit ersichtlich, haben aber auch in Folge der Unterzeichnung der Stiftung Preußischer Kulturbesitz weiterhin ausschließlich Wissenschafts- und Forschungseinrichtungen unterschrieben. ${ }^{26}$ Das, obwohl die Stiftung zusammen mit der Unterzeichnung die Freiheiten bei der Weiterverwendung des frei zugänglich gemachten Kulturerbes durch eine best practice-Erklärung im Hinblick auf die Lizenzierung eingeschränkt, und damit Sorgen und Vorurteilen aus dem Kulturbereich entgegengewirkt hat. Die Begrenzung der honorarfreien Nutzung auf nicht-kommerzielle Nutzungen entspricht dem üblichen Standard. Sie verhindert jedoch die wünschenswerte Bebilderung von Wikipedia-Artikeln, denn da die Wikipedia auf die Einnahme von Spenden angewiesen ist, agiert sie zumindest mittelbar kommerziell.

\subsection{Zugang und Weiterverwendung von Informationen zu Inhalten (Metadaten)}

Während sich die Kultur- und Wissenschaftseinrichtungen mit der freien und unbeschränkten Zurverfügungstellung von Inhalten selbst, in vielen Fällen gar nicht in ihrer Sphäre liegenden Gründen, schwer tun, dient schon die Erstellung von Metadaten dem Zugang und stehen diese darüber hinaus fast ausschließlich frei lizenziert zur Verfügung. Die Betrachtung des Zugangs und der Gestattung zur Weiterverwendung von Informationen zu Inhalten (Metadaten), soll im Folgenden für die unterschiedlichen Sparten Bibliothek, Archiv und Museum getrennt erfolgen.

\subsubsection{Bibliothek}

Als Musterbeispiel aus dem Bibliotheksbereich lässt sich die Deutsche Nationalbibliothek (DNB) anführen. Dort wurde in 2012 entschieden, die bibliografischen Daten (die Hinweise zu den Medien in der DNB, also vor allem schriftlichen Publikationen enthalten) und die Normdaten (die Hinweise zu eingebetteten Entitäten wie Orten und Personen enthalten) schrittweise für die Allgemeinheit frei lizenziert und kostenlos zur Verfügung zu stellen.

25 H.-P. Frentz, Freier Zugang zu digitalisiertem Kulturerbe für Wissenschaft und Bildung, in: P. Klimpel / E. Euler (Hrsg.), Der Vergangenheit eine Zukunft, 2015, $250 \mathrm{f}$.

26 Stand vom 15.04.2015 unter: http://openaccess.mpg.de/3883/Signatories 
Die Entscheidung beruhte auf der Erkenntnis, dass der Markt für Bibliotheksdaten am wegbrechen war. ${ }^{27}$ Nachdem absehbar war, dass mit der Umsetzung der PSI-Richtlinie im IWG und den Informationszugangsgesetzen die Grundlage für das Geschäftsmodell mit den Daten entfallen würde, sollte es dem Markt überlassen bleiben, neue Anwendungen auf der Grundlage der Daten zu entwickeln und allein die Möglichkeit, dass solche Entwicklungen und Nutzungen der Daten stattfinden könnten, rechtfertigte aus Sicht der DNB die Freigabe der Daten. ${ }^{28}$ Auch der globale Bibliotheksverbund Online Computer Library Center (OCLC), Betreiber der weltweit größten bibliografischen Datenbank WorldCat, empfiehlt eine offene Lizenzierung der Daten. ${ }^{29}$

\subsubsection{Archiv}

Neben den Bibliotheken haben auch die Archive früh die Notwendigkeit erkannt, ihre Daten der Allgemeinheit vollumfänglich zur Nutzung und Weiterverwendung zur Verfügung zu stellen. In einem von der DFG gefördertem Vorhaben werden seit Oktober 2012 archivische Erschließungsleistungen für die Nutzung, und über die Programmierschnittstelle der DDB auch zur Nachnutzung, bereitgestellt. ${ }^{30}$

\subsubsection{Museum}

Nicht alle Kultur- und Wissenschaftseinrichtungen sind so freigiebig mit ihren Daten. Dominiert von den Unterhaltsträgern, beherrscht in einigen Fällen die Angst, sich einer Einnahmequelle zu begeben, die Debatte und es wird oftmals gerade die Möglichkeit externer Effekte, also die Möglichkeit, dass Dritte mit den kosten- und zeitintensiv erstellten Datenschätzen Gewinne erwirtschaften könnten, und Gewinne privatisiert werden könnten, als kritisch eingestuft. Museen behalten auch gerne die Hoheit über die durch sie verantworteten Datenschätze, um Entstellungen die mit ihnen in Verbindung gebracht werden könnten, zu verhindern und um sicherzustel-

27 In anderen Ländern hatten bereits wichtige Nationalbibliotheken (z. B. die Britisch Library und die Nationalbibliothek in Spanien) ihre Daten der Allgemeinheit frei lizenziert zur Verfügung gestellt. 28 Siehe Lars G. Svensson, Licensing Library and Authority Data Under CCO: The DNB Experience, S. 4, abrufbar unter: http://www.google.de/url?sa=t\&rct=j\&q=\&esrc=s\&source=web\&cd=1\&ved=0CCUQFjAA\&url=http\%3A\%2F\%2Fwww.w3.org\%2F2013\%2F04\%2Fodw\%2Fodw13_submission_57. pdf\&ei=j3AuVcDSEcKrsgGxjIDQAw\&usg=AFQjCNFQ5J_7uPHsHcm_OcnuRs4WtNVmpw\&bvm=bv.90790515,d.bGg\&cad=rja (letzter Abruf 30. April 2015).

$29 \mathrm{http} / / /$ www.oclc.org/de-DE/worldcat/community/record-use/data-licensing/questions.html (letzer Aufruf 15. April 2015).

30 Archivportal-D als Teilprojekt der Deutschen Digitalen Bibliothek. Die Verknüpfung mit der Deutschen Digitalen Bibliothek ermöglicht es die archivischen Informationen mit Informationen aus Bibliotheken, Museen und anderen deutschen Kultur- und Wissenschaftseinrichtungen zu vernetzten. Siehe www.archivportal-d.de (letzter Abruf 30. Februar.2015). 
len, dass kritische und sensible Debatten (etwa zum Dritten Reich) auf der Grundlage richtiger Daten geführt werden. Nirgendwo sonst ist die Kontextualisierung von Informationen so wichtig, wie in den Museen.

\subsection{Zugang und Weiterverwendung von Inhalten}

Während bei den Informationen zu den Inhalten (Metadaten) die Betrachtung für die unterschiedlichen Sparten Bibliothek, Archiv und Museum getrennt erfolgte, soll die Betrachtung der Gestattung zur Weiterverwendung der Inhalte selbst, für die unterschiedlichen Medientypen erfolgen, denn alle Kultur- und Wissenschaftseinrichtungen haben die unterschiedlichsten Medientypen verfügbar. So steht z. B. eine Bibliothek nach wie vor vorrangig für Text, macht aber längst auch sogenannte non book materials zugänglich.

\subsubsection{Text}

Im Textbereich ist für den allgemeinen Nutzer nicht nur der Volltext urheberrechtlich noch geschützter Texte interessant. ${ }^{31}$ Genauso spannend ist insbesondere für die Forschung die Verfügbarkeit von historischen Drucken. Auf der Grundlage des Projekts „Zentrales Verzeichnis Digitalisierter Drucke (ZVDD)“ der DFG und deren Förderprogramm „Verteilte Digitale Forschungsbibliothek“, werden seit zwanzig Jahren retrospektiv die Nationalbibliographien für die verschiedenen Jahrhunderte digitalisiert, ${ }^{32}$ und im Internet über ein Portal mit Beschreibungs- und Erschließungsdaten recherchierbar gemacht. ${ }^{33}$ Durch Digitalisierung sollen die Drucke außerdem in die digitale Welt überführt werden. Das Portal ZVDD erfreut sich großer Beliebtheit und wird rege genutzt. Die Leistungen werden für den Nutzer kostenlos erbracht. Es geht um die Förderung der Forschung und die Ermöglichung kultureller Teilhabe über das Internet. ${ }^{34}$ Auch sonst sind keine Projekte bekannt, bei denen mit dem Informationsprodukt Text durch öffentliche Kultur- und Wissenschaftseinrichtungen Einnahmen erwirtschaftet werden. Im Gegenteil. Die Wissenschaft lebt durch die öffentliche Wahrnehmung und ist auf die Anknüpfung weiterer Wissenschaft an vorangegangene Ergebnisse angewiesen.

Studien belegen eindeutig: Je freier der Inhalt, desto größer die Wahrnehmung und Bekanntheit. ${ }^{35}$

31 Die Schutzfrist von Text endet gem. § 64 UrhG 70 Jahre nach dem Tod des Urhebers.

32 Siehe zu den verschiedenen Programmen der DFG: \# fehlt etwas!

33 Koordiniert durch die Arbeitsgemeinschaft Sammlung Deutscher Drucke. Siehe zu näheren Informationen: ag-sdd.de (letzter Aufruf 10. April 2015).

34 Finanziert durch staatliche Institutionen, DFG und Volkswagenstiftung.

35 The Open Access Advantage Considering Citation, Article Usage and Social Media Attention, X. Wang / C. Liu / W. Mao / Z. Fang, Cornell University Library Blog, DOI: 10.1007/s11192-015-1547-0 
Auch der Zugang ist schneller zu realisieren. Das ist für die Wissenschaft von ganz entscheidender Bedeutung. Denn dieser kommt es nicht mehr auf den Besitz, sondern nur noch auf den schnellen und komfortablen Zugang, mit anderen Worten den „Access“, an. Daher wird von Wissenschaftseinrichtungen stark das Open-AccessPublizieren gefördert. Letzten Endes verbunden mit der Hoffnung durch Publikationsdienstleistungen die Abhängigkeit von den Verlagen zu lockern und weg von, in den letzten Jahren stetig steigenden, Subskriptionsgebühren zu kommen. ${ }^{36}$

\subsubsection{Bild}

Digitale Abbildungen sind das wohl interessanteste Material für die Kreativindustrie, so z. B. die Nutzung zur Visualisierung im Journalismus oder zur kommerziellen Nutzung in der Werbung oder für Merchandising-Artikel. Digitale Abbildungen von Kulturgütern entstehen bei der Retrodigitalisierung von analogen Werken beziehungsweise der digitalen Ablichtung der analogen Welt. Die Kultur- und Wissenschaftseinrichtungen verfügen über eine Fülle wunderbarer Abbildungen. Vor allem die Museen und Einrichtungen der Denkmalpflege (wie z. B. die Stiftung Preußische Schlösser und Gärten), aber auch die Archive (insbesondere das Bundesarchiv) und die Bibliotheken verfügen über große Schätze an Fotografien und digitalen Abbildungen dieser Fotografien. Viele Einrichtungen lassen aufwendige Retrodigitalisierungen und digitale Abbildungen ihrer Sammlungsbestände (Skulpturen, Malerei, Sammlungen von Gebrauchsgegenständen etc.) herstellen. Diese dienen der Vermittlung bzw. Verwirklichung kultureller Teilhabe ebenso wie der eigenen kommerziellen Nutzung und Verwertung dieser Abbildungen über professionelle Bildagenturen und zur Herstellung etwa von Merchandising-Artikeln.

Ob die kommerzielle Verwertung für digitale Abbildungen in Frage kommt, hängt neben ihrer Attraktivität davon ab, ob sie urheberrechtlichen Schutz erfahren und ob die Kultur- und Wissenschaftseinrichtungen darüber hinaus den Zugang und die Abbildung des Sammlungsbestandes bzw. des Denkmals kontrollieren können, sodass nicht unberechtigt Abbildungen von Dritten angefertigt und vermarktet werden können. Da es kein „Recht am Bild der eigenen Sache“ gibt, ist eine solche Kontrolle nur über das Hausrecht und entsprechende Bedingungen für die Besichtigung durch Besucher möglich. ${ }^{37}$ Urheberrechtlicher Schutz an einer Abbildung entsteht abhängig vom angewandten Verfahren..$^{38}$ Damit der entstehende Schutz der Kultur- und Wissenschaftsein-

36 Vgl. V. Schallehn / R. Schimmer, Open Access, in: Praxishandbuch Bibliotheks-Management Band 1, S. 311 (323).

37 siehe hierzu ausführlich E. Euler, Recht am Bild der eigenen Sache? - Wie frei sind gemeinfreie Kulturgüter?, in: AfP 2009, 459 ff.

38 vgl. J. Weitzmann / P. Klimpel, Rechtliche Rahmenbedingungen für Digitalisierungsprojekte von Gedächtnisinstitutionen, Berlin 2014, 22f. abrufbar unter: http://dx.doi.org/10.12752/2.0.002.0 : Bei Fotografien unterscheidet das UrhG zwischen den als Werken geschützten Lichtbildwerken ( $₹$ 5, Abs. 1 , 
richtung zufällt, ist auf entsprechende Ausgestaltung der Verträge mit einem hierfür engagierten Fotografen bzw. der Mitarbeitenden zu achten. ${ }^{39}$

Auf der Grundlage des urheberrechtlichen Schutzrechts können die Kultur- und Wissenschaftseinrichtungen die Gestattung zur Weiterverwendung der Abbildungen nach gegenwärtigem Stand der Umsetzung der PSI-Richtlinie in das IWG, auch weiterhin von einer Gebühr abhängig machen bzw. müssen diese nicht gestatten. Es wird in Zukunft daher entscheidend darauf ankommen, ob an den hergestellten digitalen Abbildungen durch die Digitalisierung ein Schutzrecht entsteht, oder nicht. ${ }^{40}$

Voraussetzung für die weitere Vermarktung ist, dass die Gebühren „marktüblich“ sein müssen. Für Abbildungen kann die Marktüblichkeit der Vergütungsansätze sehr gut mit der jährlich von den Fotografieverbänden gemeinsam herausgegebenen „Übersicht der marktüblichen Vergütungen für Bildnutzungsrechte“ - der so genannten MFM-Liste - belegt werden.

In der Regel erfolgt die Vermarktung bei großen Einrichtungen über eigene Portale (z. B. Bundesarchiv) oder aber über professionelle Bildagenturen. Für den kulturellen Sektor hat sich hier insbesondere die Bildagentur bpk (Bildagentur für Kunst, Kultur und Geschichte) bei der Stiftung Preußischer Kulturbesitz etabliert.

Völlig neue Wege geht seit 2012 das Rijksmuseum in Amsterdam und stellt auf einer sehr nutzerfreundlichen Webseite eine umfangreiche Sammlung digitaler

Nr. 5 UrhG) und den sogenannten Lichtbildern (\$72 UrhG). Als Voraussetzung für den urheberrechtlichen Schutz einer Fotografie als Lichtbildwerk genügt das Vorhandensein einer persönlichen geistigen Schöpfung, die sich durch Individualität und einen erkennbaren Gestaltungswillen ausdrückt. Nach Nordemann / Vinck sind Fotografien dann als Lichtbildwerke einzustufen, wenn sie einem Fotografen insoweit persönlich zugeordnet werden können, dass sich sagen lässt, ein anderer Fotograf hätte das Foto möglicherweise anders gestaltet, indem er z. B. einen anderen Ausschnitt, Blickwinkel oder eine andere Belichtung gewählt oder einen anderen Moment festgehalten hätte, vgl. Fromm/ Nordemann, Urheberrecht, 9. Auflage, § 2 Rdn. 74. Fotografien von dreidimensionalen Werken sind im Regelfall als Lichtbildwerke einzustufen. Das gilt auch für Amateurfotos und Schnappschüsse, so dass für den einfachen Schutz als Lichtbilder nur technische Fotos, bei denen jeder Fotograf mit den gleichen Fähigkeiten und Kenntnissen das gleiche Ergebnis einer einwandfreien Wiedergabe erzielt, übrig bleiben. Dazu zählen z. B. Reproduktionen von Bildern, Fotografien (Bild vom Bild). Nach verbreiteter Ansicht entsteht ein Lichtbildrecht allerdings nur auf der ersten Stufe der Reproduktion, also bei der Reproduktion, die direkt vom Original erfolgt. Der urheberrechtliche Schutz von Lichtbildwerken besteht bis 70 Jahre nach dem Tod des Urhebers ( $\$ 64$ UrhG), der von Lichtbildern bis 50 Jahre nach Erscheinen des Lichtbildes ( 72 Abs. 3 UrhG).

39 Vgl. H.-P. Frentz, Fotorecht im Museum, in: Museumsbund (Hrsg.) Museumskunde, Band 74, $1 / 2009$.

40 siehe FN 37. Kein Schutz entsteht für die Retrodigitalisierung bei der Massendigitalisierung im Scanverfahren bzw. allgemein dann, wenn alle Einflussfaktoren für die digitale Abbildung weitgehend maschinell und automatisch gewählt werden und es an der spezifisch fotografischen Leistung fehlt, die ein entspr. Leistungsschutzrecht rechtfertigt. War die Vorlage gemeinfrei, gilt das somit auch für das digitale Abbild. Da zunehmend auch dreidimensionale Werke durch Digitalisierungsstraßen geschickt werden, einem Verfahren, bei dem alle Einstellungen vollautomatisiert ablaufen, bleibt abzuwarten, wie sich die Rechtsprechung entwickelt. 
Werkabbildungen in hoher Auflösung zu jeder Art der Nutzung, auch der kommerziellen, honorarfrei bereit. ${ }^{41}$ Das Rijksmuseum sah sich einer öffentlichen Diskussion über die staatliche Finanzierung ausgesetzt, wo ein Großteil der gesammelten und mit Steuergeldern finanzierten Werke aus Platzgründen nicht gezeigt werden könne. Die Auseinandersetzung wurde besonders hitzig geführt, als das Museum während Umbaumaßnahmen überhaupt keine Werke zeigen konnte. Die Überlegungen, wie sich die Werke in Gesamtheit zeigen lassen könnten, haben zum Internet geführt.

Im Internet können alle Werke, auch der Depotbestand, ohne Platzprobleme gezeigt werden. Während der Service am Anfang noch eingeschränkt war und die Werke unfrei lizenziert waren, kam das Museum über die Zeit zu der Einsicht, dass die unfreie Lizenzierung nicht vor unberechtigten Nutzungen schützt, aber berechtigte Nutzungen ungewollt verhindert. Daher wurde entschieden, alle Werke in die public domain und damit für alle Nutzungen frei zu geben. Die Finanzierung dieses Services basiert auf Sponsoring. Vielen scheint das Angebot so gut zu gefallen, dass sie bereit sind, die Politik des Museums zu unterstützen. Tatsächlich sind die Einnahmen nach Umstellung des Geschäftsmodells viel höher, als die Einnahmen aus der kommerziellen Vermarktung je gewesen sind.$^{42}$ Die freie Zurverfügungstellung der Werke über das Internet hilft, das Original im Gedächtnis lebendig zu halten und animiert dazu, das Original zu besuchen, mit anderen Worten: schafft Öffentlichkeit. ${ }^{43}$ Weitere angesehene Einrichtungen sind dem Beispiel des Rijksmuseums gefolgt, so das Amsterdam Museum, Hewitt Cooper New York, Los Angeles County Museum, die Nationalarchive der Niederlande und Finnland, Armoury Stockholm, das Getty Institut und SMK Dänemark. ${ }^{44}$ Jeweils war entscheidend, dass festgestellt wurde, dass nach

41 Rijksstudio unter: www.rijksmuseum.nl (letzter Aufruf 10. April 2015). Zu den Hintergründen und der Strategie der Umstellung siehe: J. Pekel, Democratizing the Rijksmuseum, abrufbar unter: http://pro.europeana.eu/files/Europeana_Professional/Publications/Democratising\%20the\%20Rijksmuseum.pdf (letzter Aufruf 20. April 2015).

42 Lizzy Jongma, Datenmanagerin beim Rijksmuseum auf Nachfrage: „We never charged a lot of money for our images. But this money never covered the costs of selling images: we had very old systems and a lot of bureaucracy so even though it generated money we also had to pay staff etc to cash the revenue. Our experience is that we don't miss income by not selling images. And someone else is not making money with our collection either. Some of our icons are used for commercials etc. but no one runs a business on our collection. And most commercials generate attention for the Rijksmuseum and helps people understand what the museum is about. So it's a lot of free publicity. And currently we get project funding from private and government funds to digitize images and collections: funders are very happy when you share the results of their projects with the rest of the world! So instead of selling 1 image for 40,- euro's and waiting until we have enough money to start a new digitization project we now get project funding to digitize collections. We are even speeding up our digitzation effords. We planned to digitize the entire collection in 13 years but we are working on reducing that to 5 years. “

43 M. Sanderhoff (Hrsg.), Sharing is Caring, Openess and Sharing in the cultural heritage sector, abrufbar unter: http://www.smk.dk/en/about-smk/smks-publications/sharing-is-caring/read-sharing-is-caring/ (letzter Aufruf: 20. April 2015).

44 Wieder weitere, wie das Metropolitan, sind kurz davor umzustellen. 
Abzug aller Kosten für die Vermarktung durch Einräumung einfacher Nutzungsrechte (Personal, Software, Abrechnung, Werbung, Rechtsverfolgung, Rechteklärung und Management etc.) tatsächlich keine nennenswerten Einnahmen generiert wurden, die den Aufwand gerechtfertigt hätten.

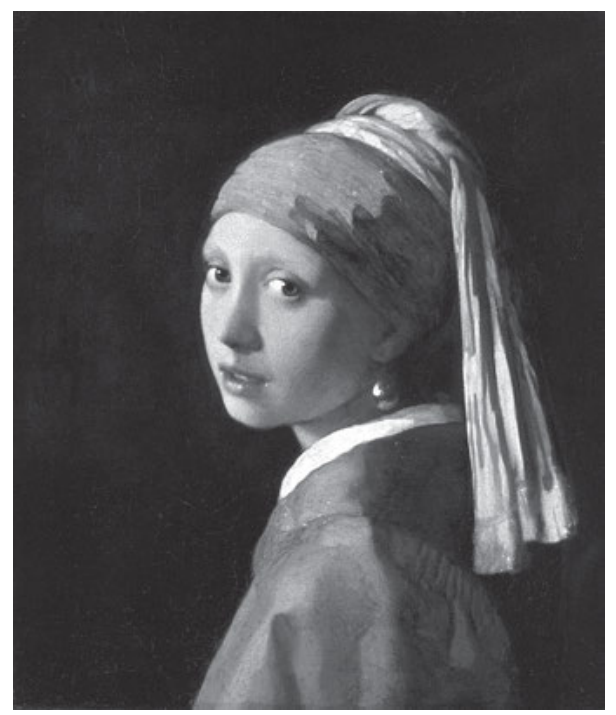

Abb. 2: Girl with a Pearl Earring von Johannes Vermeer - www.geheugenvannederland.nl : Home : Info : Pic. Lizenziert unter Gemeinfrei über Wikimedia Commons - http://commons.wikimedia.org/ wiki/File:Girl_with_a_Pearl_Earring.jpg\#/media/File:Girl_with_a_Pearl_Earring.jpg

\subsubsection{Audio und audiovisuelles Material}

Über Audio und audiovisuelles Material verfügen vor allem die Mediatheken und Rundfunkarchive. Die Deutsche Kinemathek in Berlin etwa verfügt über einige tausend Filme. Diese können aus dem Archiv zu wissenschaftlichen Zwecken am Schneidetisch oder im hauseigenen kleinen Kino angesehen werden. Man kann sie auch für den nichtkommerziellen Einsatz ausleihen. Der kommerzielle Einsatz bedarf gesonderter Absprachen und einer Vergütung, generiert jedoch keine wesentlichen Einnahmen. Der laufende Betrieb der Stiftung des bürgerlichen Rechts wird über Zustiftungen des Bundes finanziert. 


\section{Open-Access-Geschäftsmodelle}

Auch wenn die Kultur- und Wissenschaftseinrichtungen selbst nach Umsetzung der PSI-Richtlinie im IWG nicht verpflichtet sind herkömmliche Geschäftsmodelle zugunsten von Open Access Geschäftsmodellen zu revidieren bzw. sie bereits gegenwärtig ein Maximum an Mitteln und Kapazitäten einsetzen, um der Verpflichtung, kulturelle Teilhabe über digitale und vernetzte Medien und das Internet zu verwirklichen, nachzukommen, lohnt sich dennoch bzw. gerade dann, eine Auseinandersetzung mit den Möglichkeiten die Open-Access-Geschäftsmodelle bieten. Die sogenannten OpenBusiness- Modelle sind Geschäftsmodelle, die auf freien Inhalten beruhen und bei denen Umsatz durch eine am Produkt orientierte Serviceleistung erzielt wird. Sie sind durchlässiger für die Bedürfnisse der Kunden und setzen stärker auf Partnerschaften mit anderen Einrichtungen, als in klassischen Modellen üblich.

Obwohl öffentliche Information als treibende Kraft bei der Förderung von Transparenz und der Wissensgesellschaft eingestuft wird, ist die Kenntnis der Geschäftsmöglichkeiten, die sich auftun, indem öffentliche Information in neue oder bestehende Produkt- und Dienstleistungen eingebaut wird bzw. diese unterstützt, gering. In einer umfassenden Studie, die sowohl die verschiedenen Player als auch die verschiedenen Herangehensweisen betrachtet, haben Ferro und Osella mögliche archetypische Geschäftsmodelle auf der Basis öffentlicher Information entwickelt. ${ }^{45}$ Die Untersuchung betrachtet auch mögliche Geschäftsmodelle für Kulturportale, also auf den Angeboten, die aus den Einrichtungen kommen, aufbauende vermittelnde Angebote. ${ }^{46}$

Welche der Geschäftsmodelle sich im Einzelnen anbieten, ist abhängig davon, an welcher Schnittstelle die jeweilige Kultur- und Wissenschaftseinrichtung agiert und ob es sich bei dem Rohstoff um öffentliche Information handelt. In der von Ferro und Osella dargestellten Reinform wird in den seltensten Fällen agiert werden. Oft werden Mischformen nötig sein. Das hängt schon damit zusammen, dass in vielen Fällen die Einrichtung nicht vollständig über die Rechte am Ausgangsmaterial verfügt, sondern nur abgeleitete Rechte besitzt. Rechte mussten vom ursprünglichen Rechteinhaber bzw. Rechteinhaber an der Digitalisierungsvorlage entweder erworben werden, oder aber es besteht dauerhaft die Notwendigkeit, den ursprünglichen Rechteinhaber an der Verwertung zu partizipieren. In diesen Fällen ist eine vollständige Umstellung des Geschäftsmodells, wie etwa beim Rijksmuseum, nicht möglich.

Grundsätzlich werden für Portal- bzw. Plattformlösungen, die mit freien Inhalten arbeiten, drei Open-Access-Modelle unterschieden: ${ }^{47}$

45 Ferro, E. / Osella, M.: Eight Business Model Archetypes for PSI Re-Use, April 2013 abrufbar unter: www.w3.org/2013/04/odw/odw13_submission_27.pdf (letzter Abruf am 30. März 2015).

46 Hierzu siehe auch den Beitrag in diesem Buch von H. Simon.

47 S. Seitz, Geld verdienen mit freier Bildung in: P. Otto (Hrsg.), Das Netz, Berlin 2015, 98. 
Beim „Freemium-Modell“ werden Inhalte unter einer freien Lizenz zugänglich gemacht, ohne, dass hierfür eine Gebühr zu entrichten ist. Die Lizenz verbietet allerdings in der Regel die kommerzielle Nutzung und verlangt die Nennung des Rechteinhabers bei der weiteren öffentlichen Verwendung. ${ }^{48}$ Dieses Modell dient der Erhöhung des Bekanntheitsgrades und ist im weitesten Sinne eine Marketingmaßnahme.

Beim „Efficiency-Modell“ werden über (hauptsächlich vom Staat) finanzierte beziehungsweise geförderte Plattformen Inhalte so frei wie möglich zur Verfügung gestellt, um in einem kostenträchtigen Bereich (etwa der kulturellen Daseinsfürsorge) Kosten einzusparen. So soll die Effektivität und Effizienz in einem System gesteigert werden und Unabhängigkeit von kommerziellen Anbietern (etwa. STM Verlagen) erreicht werden.

Beim „Subsidizing-Modell“ werden Inhalte über Plattformen wie oben bereitgestellt, allerdings steht hier nicht die Kostensenkung und Unabhängigkeit von kommerziellen Anbietern im Vordergrund, sondern die Ermöglichung kultureller Teilhabe bei Wahrung der Chancengleichheit.

Hierauf aufbauend sind ergänzende Leistungen denkbar:

Etwa lassen sich durch Intermediäre Einnahmen erzielen, indem der Zugang bzw. die Verwendbarkeit von Material für Entwickler oder Wissenschaftler erleichtert wird. Das Wertversprechen hängt von einem attraktiven und wenig kostenintensivem Initialangebot ab, das dazu ermutigt oder motiviert, weitere Käufe oder Zusatzleistungen in Anspruch zu nehmen, wobei für diese bei starrer Nachfragkurve hohe Margen in Ansatz gebracht werden können. So werden etwa Daten / Inhalte kostenlos in CloudComputing-Technologie gehostet und sind für Jedermann über freie Programmierschnittstellen zugänglich, während die Rechnerleistung, die wie eine Dienstleistung für den Aufruf aufgewandt wird, in Rechnung gestellt wird. Dieses Modell funktioniert natürlich nur in Zusammenhängen, bei denen die rechenintensiven Kosten signifikant sind.

Auch lassen sich durch Intermediäre Einnahmen erzielen, die den Zugang bzw. die Verwendbarkeit von Material für Entwickler erleichtern, wobei dieses auf proprietären, sehr zuverlässigen Servern liegt. Durch die Aggregation von verschiedenen Inhalten, deren Standardisierung und Strukturierung, lassen sich sehr genau unterschiedliche Anfragen beantworten. Der Nutzer erhält einen full service und muss sich nicht mit verschiedenen Suchmasken auseinandersetzen. Das macht das Angebot für ihn als one stop shop attraktiv. Dieser Ansatz ist entscheidend, um Kosten und insbesondere die Transaktionskosten zu senken. Je besser das gemachte Angebot, desto größer die Nachfrage, die auch den Preis für das Angebot und damit die Einnahmemöglichkeiten definiert.

Auch ist möglich, dass nicht die Entwickler zur Kasse gebeten werden, sondern diejenigen, die verpflichtet sind, Informationen für Erstere bereitzustellen (etwa nach IWG). Dieses Modell ist für beide Seiten gewinnbringend. Auf Entwicklerseite sind die

48 So z. B. die Creative-Commons-Lizenz attribution, non-commercial. 
Hindernisse überwunden (z. B. können Daten / Informationen ohne Transaktionskosten abgerufen werden). Auf Seiten der Informationsanbieter haben diese Zusatznutzen etwa durch schnellen Upload von Datensets öffentlicher Bereitsteller, Standardisierung von Formaten, Unterfütterung mit Metadaten und automatische externe Anzeige der offenen Daten über Programmierschnittstellen und grafische Benutzeroberflächen. Angebotene Dienste können z. B. das Hosting, die Metadatenpflege, die Einhaltung von Standards und Formaten und deren Kontrolle oder aber die Bereitstellung von Werkzeugen sein.

Öffentliche Stellen, die ein solches Angebot machen, gehen langfristige Beziehungen mit Providern ein und zahlen eine periodische Gebühr, die vom Grad der Leistungen des Providers ebenso abhängt, wie von anderen technischen Parametern.

Im Platforming-Modell sind die „Kunden“ zugleich die „Produzenten der Inhalte“. Der Pflege der Kunden, durch die die Plattform überhaupt erst lebt, kommt daher eine erhebliche Bedeutung zu. Sie ist eines der zentralen Elemente für das Funktionieren des Modells.

\section{Ausblick und Resümee}

Open Access ist zum Teil eine Verpflichtung der Einrichtungen, die ihrem Auftrag im 21. Jahrhundert, dem digitalen und vernetzten Jahrhundert, auch über das Internet nachkommen müssen, zum Teil eine Selbstverpflichtung. Open Access kann ein vielversprechendes Geschäftsmodell sein. Eine einheitliche Best-Practice-Linie gibt es noch nicht. Diese sollte den Auftrag der Kultur- und Wissenschaftseinrichtungen in den Vordergrund rücken und mit den Unterhaltsträgern dieser Einrichtungen abgestimmt sein. Dabei sollte Berücksichtigung finden, dass optimale Vernetzung sich nur mit offenen Daten Linked-Open-Data (LOD) realisieren lässt und nur mithilfe von LOD aus Information Wissen wird. ${ }^{49}$ Letzten Endes können nur die Kultur- und Wissenschaftseinrichtungen unser kulturelles Erbe digitalisieren und online für alle verfügbar machen - mit einer wissenschaftlichen Präzision, die Google fehlt. Ein Wissenschaftler hat es folgendermaßen ausgedrückt: Texte sind lebendige Wesen: Digitalisierung verwandelt sie von Raupen in Schmetterlinge. Aber die Digitalisierung verspricht uns nicht bloß bessere Webseiten, sondern auch die Verwandlung humanistischer Bildung, die man im Englischen als humanities bezeichnet, in jene Wissenschaft, die im Englischen science genannt wird, damit ist die Verwendung von Zahlen gemeint, um Hypothesen zu überprüfen und nicht nur „anekdotisch“ mit Zitaten zu belegen. ${ }^{50}$ Mithilfe von Datenanalyseverfahren können auch die Geisteswissenschaf-

49 Mittelbach SUB Göttingen

50 A. M. Leroi, Cicero zählen, Algorithmus oder Kritik? Plädoyer für eine universelle Kulturtheorie, in: Süddeutsche Zeitung vom 6. März 2015, 11. 
ten ganz neue Forschungsfragen stellen und es lassen sich auch hier die Erkenntnisse auf ganz neue Weise, etwa mit Hilfe visueller Verfahren, darstellen. ${ }^{51}$

Den Kultur- und Wissenschaftseinrichtungen ist zu raten, sich nicht verunsichern $\mathrm{zu}$ lassen. Im Rahmen eines Strategie- und Selbstverwirklichungsprozesses sollten sie ermitteln, wie sich die eigene Vision und Mission am besten verwirklichen lässt und welche Geschäftsmodelle am besten zum Auftrag passen. Es ist Sache der Einrichtungsleitung, im Sinne von echtem „Leadership“ innovative Ideen umzusetzen und dabei auch einmal zu riskieren, von klassischen Wegen abzugehen. Dabei muss Innovation nicht bedeuten, das Rad jedes Mal neu zu erfinden. Konzentration und Kooperation sind der Schlüssel zu vielversprechenden Angeboten. Insbesondere die DDB bietet sich als Kooperationspartner für digitale Strategien der Vermittlung von Kultur und Wissen an.

51 S. Herrmann, Leuchtfeuer der Kulturgeschichte, Beginnt endlich die Digitalisierung der Geisteswissenschaften? Kunsthistoriker durchforsten riesige Datensätze mit Methoden der Netzwerk-Analyse, um darin entscheidende Trends aufzuspüren, in: Süddeutsche Zeitung vom 1. August 2015, 14. 